\title{
Atomic-Resolution Imaging of Fast Nanoscale Dynamics with Bright Microsecond Electron Pulses
}

\author{
Pavel K. Olshin, ${ }^{\dagger}$ Gabriele Bongiovanni, ${ }^{\dagger}$ Marcel Drabbels, and Ulrich J. Lorenz* \\ Cite This: https://dx.doi.org/10.1021/acs.nanolett.0c04184 \\ Read Online
}

\section{ACCESS | Lلw Metrics \& More | 回 Article Recommendations | si Supporting Information}

ABSTRACT: Atomic-resolution electron microscopy is a crucial tool to elucidate the structure of matter. Recently, fast electron cameras have added the time domain to high-resolution imaging, allowing static images to be acquired as movies from which sample drift can later be removed computationally and enabling real-time observations of atomic-scale dynamics on the millisecond time scale. Even higher time resolution can be achieved with short electron pulses, yet their potential for atomicresolution imaging remains unexplored. Here, we generate high-brightness microsecond electron pulses from a Schottky emitter whose current we briefly drive to near its limit. We demonstrate that drift-corrected imaging with such pulses can achieve atomic resolution in the presence of much larger amounts of drift than with a continuous electron beam. Moreover,

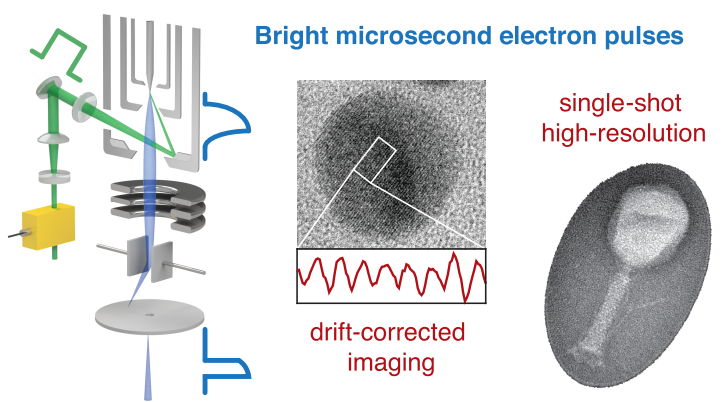
such pulses enable atomic-resolution observations on the microsecond time scale, which we employ to elucidate the crystallization pathways of individual metal nanoparticles as well as the high-temperature transformation of perovskite nanocrystals.

KEYWORDS: Time-resolved transmission electron microscopy, drift correction, single shot imaging, high-resolution TEM, perovskite nanoparticles

pecimen drift presents a fundamental challenge for high$\checkmark$ resolution imaging, particularly for in situ experiments where rapid changes of the sample temperature or some other variable induce large amounts of drift. ${ }^{1}$ But even the exposure of the sample to the electron beam itself may cause beaminduced sample motion. Fast electron cameras have alleviated this problem, allowing one to record micrographs as movies, so that drift can be removed computationally by aligning the movie frames to each other before adding them up. ${ }^{2}$ Such dose-fractionated imaging has, for example, become crucial in cryo-electron microscopy. ${ }^{2}$ Nevertheless, if the amount of drift present in a movie frame exceeds the target resolution, it has to be discarded. This is frequently the case for the first frames of cryo-electron micrographs, which are particularly affected by beam-induced drift. ${ }^{2}$

Fast electron cameras are also used to observe processes in real time that are purposefully induced in situ, for example, with electrical impulses, through mechanical deformation, heating, or laser irradiation of the sample. ${ }^{3-7}$ The time resolution of such in situ experiments is ultimately limited by the frame rate of the electron camera, as is the amount of drift that can be removed with dose-fractionated imaging. A vastly higher time resolution can be obtained by imaging with short electron pulses, a principle that is employed in time-resolved electron microscopy to capture processes down to the femtosecond time scale. $^{8-12}$ However, atomic-resolution imaging with short electron pulses has largely remained elusive. For example, while it has been proposed that it should be possible to image the dynamics of proteins at atomic resolution with a single electron pulse, ${ }^{13,14}$ the spatial resolution currently achieved in such single-shot experiments is several nanometers. ${ }^{14}$ The challenge here lies in the creation of electron pulses that are coherent enough to enable atomicresolution imaging but also contain a sufficiently large number of electrons to be able to capture the image information in a single shot, or a small number of pulses if the image is recorded stroboscopically.

\section{RESULTS AND DISCUSSION}

Generation of High-Brightness Electron Pulses. Here, we demonstrate that such intense, high-brightness electron pulses can be generated by temporarily boosting the emission current of a Schottky emitter to near its limit ${ }^{15}$ and chopping the electron beam into pulses with an electrostatic deflector. As illustrated in Figure 1a,b, we irradiate the emitter tip with a microsecond laser pulse, which is generated by chopping the output of a continuous laser beam (532 nm) with an acoustooptic modulator. A mirror embedded within the emitter

Received: October 19, 2020

Revised: December 3, 2020 

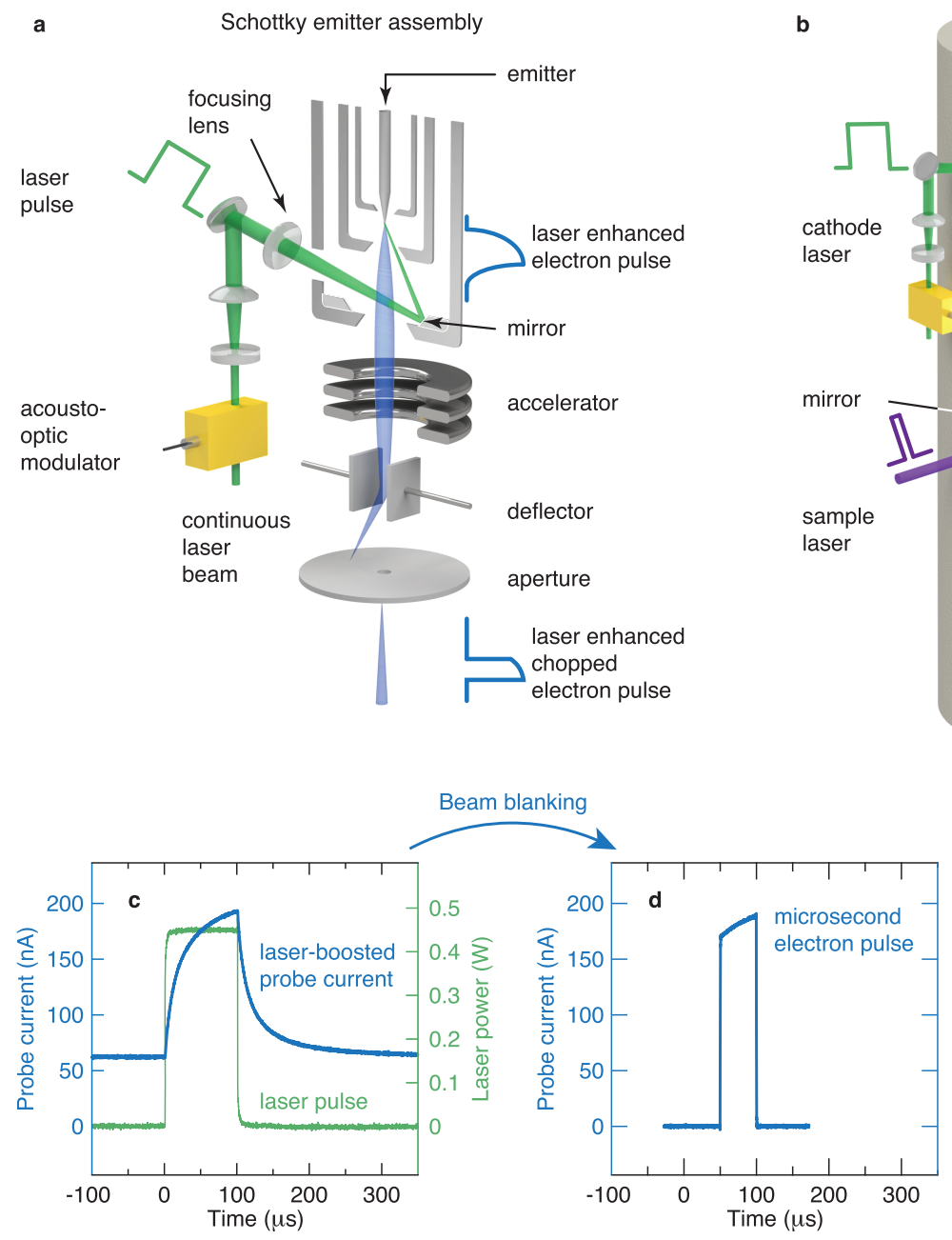
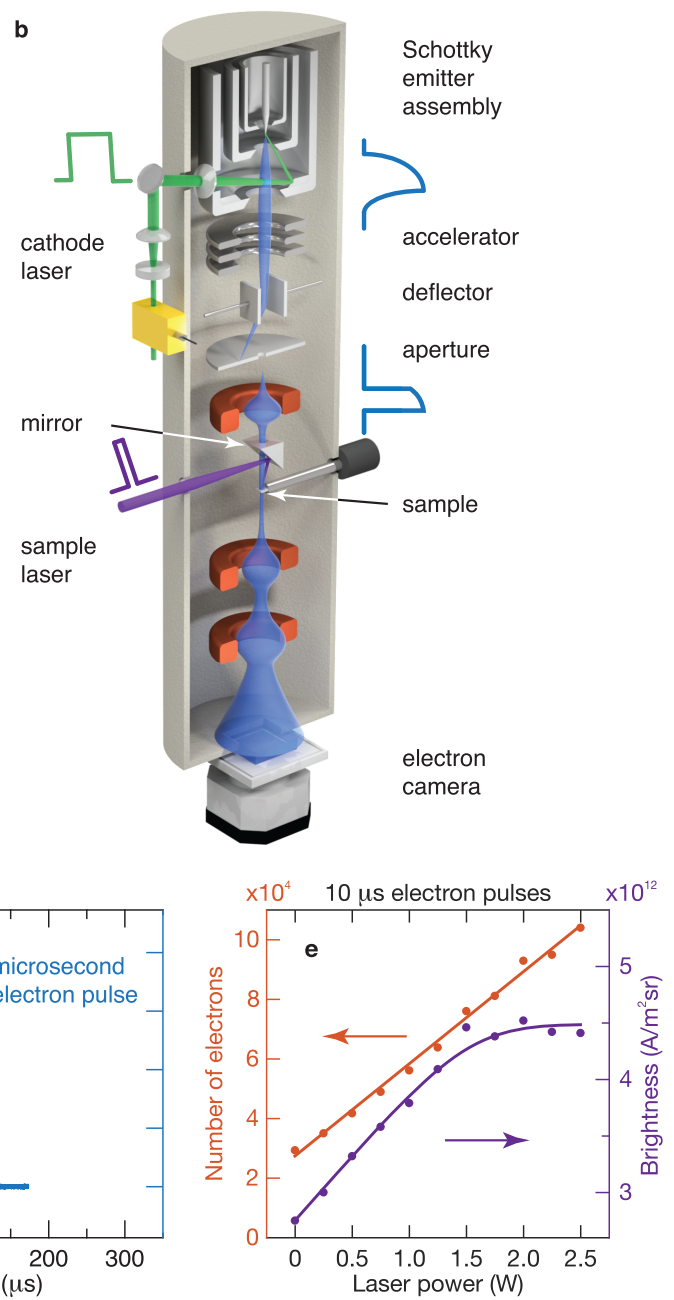

Figure 1. Generation of intense microsecond electron pulses of high brightness. (a) A microsecond laser pulse heats the tip of a Schottky emitter to boost emission, while an electrostatic deflector chops the electron beam into microsecond pulses. (b) Illustration of the time-resolved electron microscope. Dynamics of the sample are initiated with a short laser pulse and probed with a microsecond electron pulse. (c) The probe current (blue) is boosted when the emitter tip is heated with a microsecond laser pulse (green). (d) An intense microsecond electron pulse is obtained by chopping the boosted electron beam with the deflector. (e) Number of electrons in a $10 \mu$ s electron pulse (red) and instantaneous brightness (purple) as a function of laser power. The solid lines serve as a guide for the eye.

assembly directs the laser beam at the tip of the Schottky emitter (Figure 1a), where it is focused to a spot size of $17 \mu \mathrm{m}$ fwhm. As shown in Figure 1c, illumination with a $100 \mu$ s laser pulse of $0.44 \mathrm{~W}$ (green curve) temporarily boosts the probe current (blue) from $60 \mathrm{nA}$ to a peak of $200 \mathrm{nA}$. Under these conditions, the emitter tip reaches an estimated temperature of about $2500 \mathrm{~K}$, significantly above the maximum safe operating temperature of $1850 \mathrm{~K}$ (ref 16), which briefly increases the emission current to an extreme value ${ }^{15}$ that can otherwise not be sustained without damaging the emitter. ${ }^{16,17}$

As illustrated in Figure 1a, the laser-boosted electron beam is chopped into pulses by means of an electrostatic deflector that is placed after the accelerator of the gun and that sweeps the beam over a small aperture. This allows us to generate electron pulses of microsecond duration, as demonstrated in Figure 1d, and even pulses as short as $100 \mathrm{~ns}$. These laser-boosted pulses are not only intense but also possess a high instantaneous brightness, which is crucial for high-resolution imaging. ${ }^{18}$ Figure le shows that the brightness initially increases linearly with laser power but levels off as electron-electron interactions in the beam become more frequent. ${ }^{17,19}$ At the highest laser power, it reaches 1.7 times the value without boost laser, while the number of electrons per pulse has almost quadrupled (see also Figure S1). As illustrated in Figure 1b, the electron gun and deflector assembly are mounted on a JEOL 2010F transmission electron microscope that we have modified for time-resolved experiments. ${ }^{19}$ Dynamics of the specimen are initiated in situ with pulses from a $405 \mathrm{~nm}$ diode laser that is directed at the sample with a mirror mounted above the upper pole piece of the objective lens $(34 \mu \mathrm{m}$ spot size in the sample plane). A laser-boosted electron pulse is then used to capture a snapshot of the dynamics with the electron camera.

Drift Corrected Imaging. We first demonstrate the potential of our laser boosted electron pulses to improve the resolution of drift-corrected imaging by delivering the electron dose per movie frame in a short burst instead of spreading it out evenly, so that even larger amounts of drift can be outrun. Figure 2a displays a micrograph of a $15 \mathrm{~nm}$ diameter gold particle on an amorphous carbon substrate (full frame image in Figure S2) acquired with a continuous electron beam in the presence of substantial drift. In order to induce a well-defined 
Drift-corrected imaging with microsecond electron pulses Drift velocity: $9 \mathrm{~nm} / \mathrm{s}$
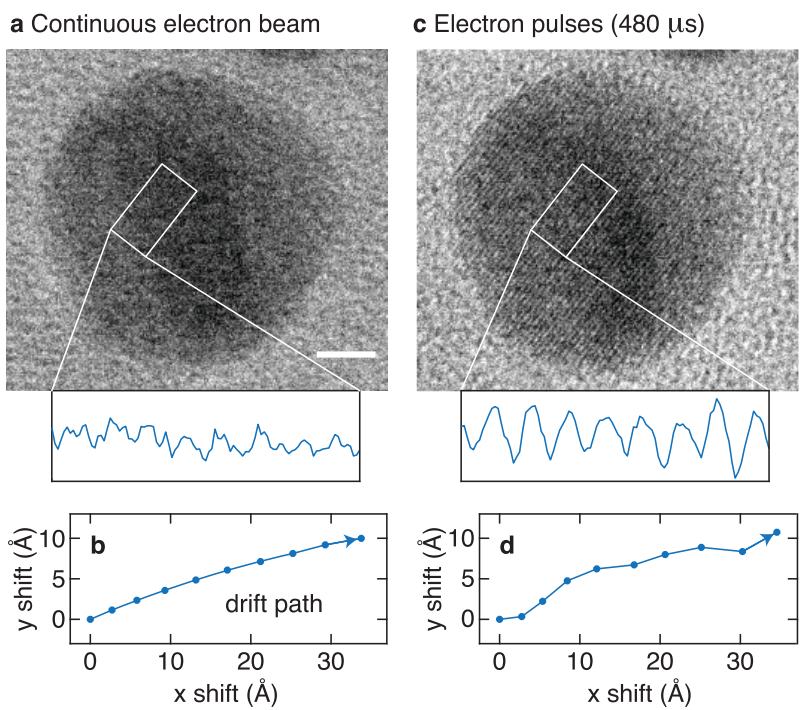

Drift velocity: $3 \mathrm{~nm} / \mathrm{s}$ e Continuous electron beam
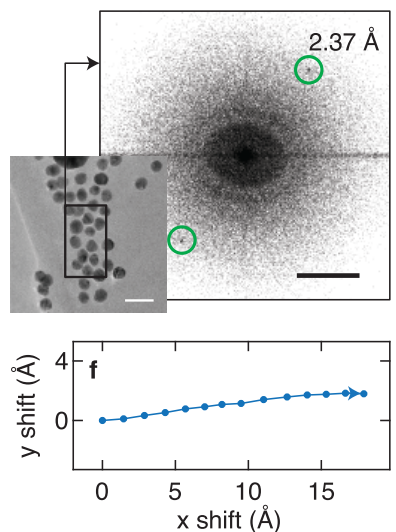

g Electron pulses (480 us)
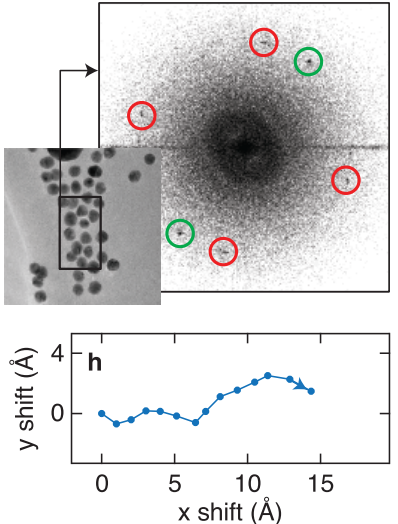

Figure 2. Drift-corrected imaging with microsecond electron pulses. $(a, c)$ Drift-corrected images of a gold nanoparticle (from the full frame micrographs in Figure S2), recorded with a continuous electron beam (a) and with $480 \mu$ s electron pulses (c) in the presence of a drift velocity of $9 \mathrm{~nm} / \mathrm{s}$. When imaged with electron pulses, the particle exhibits lattice fringes, as evident in the intensity profile of the region marked with a white rectangle. Scale bar, $3 \mathrm{~nm}$. (b,d) Drift path of the sample during acquisition of the images in (a) and (c), respectively. $(e, g)$ Drift-corrected images of an ensemble of gold nanoparticles, recorded with a continuous electron beam (e) and with $480 \mu \mathrm{s}$ electron pulses $(\mathrm{g})$ in the presence of a drift velocity of $3 \mathrm{~nm} / \mathrm{s}$. Diffractograms of the area marked with a black rectangle reveal several reflections of the gold nanoparticles $(2.37 \AA)$ that are present in the image recorded with a pulsed electron beam but absent when recorded with a continuous beam. Scale bars, $30 \mathrm{~nm}$ and $2.5 \mathrm{~nm}^{-1}$. $(\mathrm{f}, \mathrm{h})$ Drift path of the sample during acquisition of the images in (e) and $(\mathrm{g})$, respectively.

amount of drift, the sample was irradiated with a continuous laser beam whose power was increased linearly during acquisition. The image was recorded as a movie of 10 frames of $41 \mathrm{~ms}$ duration each, corresponding to the fastest frame rate of our camera at full frame read-out $(410 \mathrm{~ms}$ total exposure time), and subsequently drift corrected with cisTEM. ${ }^{20}$ The drift path obtained in the alignment procedure reveals that the sample drifted with a speed of $9 \mathrm{~nm} / \mathrm{s}$, corresponding to $3.5 \AA$ per frame (Figure $2 \mathrm{~b}$ ), which explains the blurry appearance of the image. In contrast, if we acquire each movie frame with a laser-boosted electron pulse of $480 \mu \mathrm{s}$ duration, the image resolution is markedly improved, as shown in Figure 2c (same total electron dose and drift, see Figure $2 \mathrm{~d}$ ). The gold nanoparticle now exhibits lattice fringes, which are also evident in the intensity profile (inset) of the area marked with a rectangle and which are entirely absent in the image recorded with a continuous electron beam.

Imaging with intense microsecond electron pulses affords an improved resolution even at lower drift rates, similar, for example, to those encountered in the first movie frames of cryo-electron micrographs. ${ }^{2}$ When we image an ensemble of gold nanoparticles on lacey carbon with 14 frames of $41 \mathrm{~ms}$ duration (Figure 2e,g,) while the sample drifts with a speed of $3 \mathrm{~nm} / \mathrm{s}$, corresponding to $1.4 \AA$ per frame (Figure 2f,h), diffractograms of the area marked with a rectangle reveal several reflections (red circles, $2.37 \AA$ ) for the micrograph recorded with pulsed illumination (Figure $2 \mathrm{~g}$ ) that are absent or much weaker with a continuous beam (Figure 2e). As we demonstrate in Figure S3, imaging with electron pulses is also advantageous if drift is not induced by an external stimulus but instead results from the exposure of the sample to the electron beam itself.

Single-Shot Imaging. Next, we demonstrate the ability of our intense electron pulses to capture microsecond single-shot images with atomic resolution. Figure 3a displays a $15 \mathrm{~nm}$ diameter gold nanoparticle that was imaged with 15 laserboosted electron pulses of $10 \mu \mathrm{s}$ duration. In order to obtain the highest electron dose, the electron beam is almost fully converged to an area barely larger than the object. Even under such extreme imaging conditions, the particle features lattice fringes, which are also evident in the intensity profile of the area marked with a rectangle as well as the diffractogram of the image (insets). An individual single-shot micrograph is shown in Figure 3b. Even though it is noisier, it demonstrates that atomic-resolution information can be captured with just a single $10 \mu$ s electron pulse. Another example is presented in Figure 3c, which shows a micrograph of negatively stained Bacteriophage T4 (ref 21), imaged with 20 pulses of $50 \mu \mathrm{s}$ duration. An intensity profile and diffractogram (insets) of the phage tail (white bracket) reveal the characteristic $39 \AA$ spacing of the tail sheath proteins, ${ }^{21}$ which are also evident in an individual single-shot image (Figure $3 \mathrm{~d}$ ). We conclude that pulses of tens of microseconds offer sufficient contrast for single-shot imaging while being coherent enough to achieve atomic resolution.

Single-Shot Imaging of Nanoscale Dynamics. Finally, we demonstrate that our laser-boosted electron pulses allow us to capture fast, atomic-scale transformations in a single shot. We illustrate this by studying the competing crystallization pathways of an individual lead nanoparticle (Figure $4 \mathrm{a}$, circle), which we melt in situ with a laser pulse $(500 \mu \mathrm{s}, 1 \mathrm{~mW})$ before letting it rapidly crystallize in the presence of an adjacent $\mathrm{Cs} \mathrm{PbBr}_{3}$ perovskite nanocrystal (rectangle) that provides a nucleation seed. A single-shot image recorded during the laser pulse (Figure 4b) establishes that the lead particle is completely melted, while the perovskite remains crystalline (see also the diffractograms of the particles in the insets). Subsequently, the lead particle crystallizes in a new configuration with several crystalline domains (Figure 4c). 


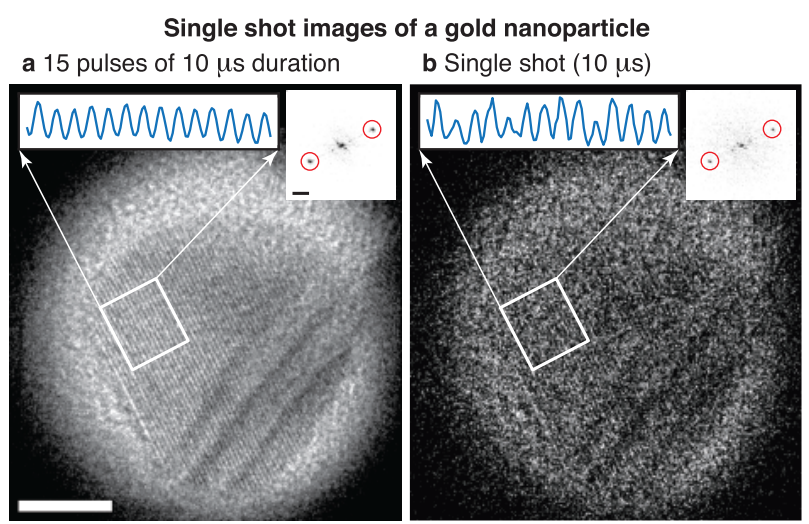

Single shot images of Bacteriophage T4 c 20 pulses of 50 us duration d Single shot $(50 \mu \mathrm{s})$
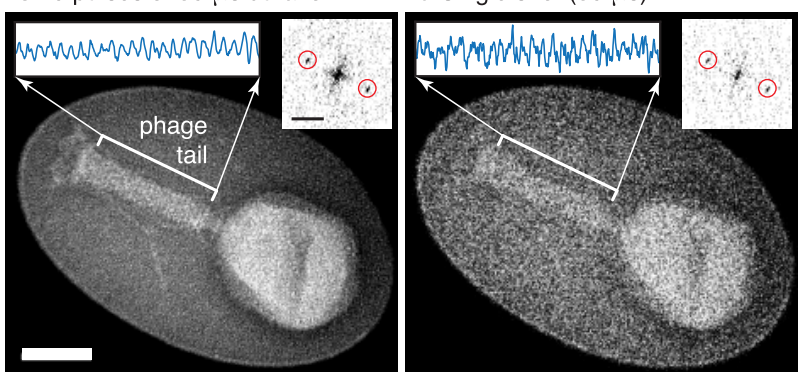

Figure 3. Atomic-resolution single-shot imaging. (a,b) Single-shot images of a gold nanoparticle recorded with $10 \mu$ s electron pulses. In (a), 15 pulses of $10 \mu$ s duration are averaged, while (b) shows an image recorded with an individual $10 \mu$ s pulse. Line profiles of the area marked with a rectangle (left insets) and diffractograms of the particle (right insets) reveal lattice fringes with a spacing of $2.35 \AA$. Scale bars, $5 \mathrm{~nm}$, as well as $2 \mathrm{~nm}^{-1}$ in the inset. (c,d) Single-shot images of negatively stained Bacteriophage T4 recorded with $50 \mu \mathrm{s}$ electron pulses. In (c), 20 pulses of $50 \mu$ s duration are averaged, while (d) shows an image recorded with an individual $50 \mu$ s pulse. Intensity profiles (left insets) and diffractograms (right insets) of the area corresponding to the phage tail (marked with a white bracket) reveal the spacing of the tail sheath proteins of $39.0 \AA$. Scale bars, $50 \mathrm{~nm}$, as well as $0.25 \mathrm{~nm}^{-1}$ in the inset.

When we repeat the crystallization process, different configurations are randomly obtained. Frequently, however, the perovskite nanocrystal appears to act as a nucleation seed (Figure S4) creating configurations such as the one in Figure $4 \mathrm{~d}$ in which the lattice fringes of the lead particle are parallel to a set of planes of the perovskite crystal. This is also evident in the diffractograms, where the corresponding sets of reflections of both particles fall onto the same line (highlighted with purple bars). The lead particle has formed a single-crystal, as evident from the purple overlay highlighting areas of the same crystal orientation that was obtained by Fourier filtering with the reflections selected that are circled in the diffractogram. Melting and recrystallization creates yet another configuration (Figure 4f), which appears to result from the competition of different crystallization pathways. While the lattice fringes visible in the top (green) are randomly oriented, those in the bottom (red) are aligned with another set of lattice planes of the adjacent perovskite crystal (red bars in the diffractograms). A snapshot of the crystallization process yields insights into the crystallization trajectory (Figure 4e). Surprisingly, formation of the randomly oriented domain (green) precedes that of the heterogeneously nucleated domain (red), which appears to be just forming in the single-shot image. Such real-time observations promise to elucidate a range of phenomena that have so far only been studied theoretically for rapidly crystallizing metal nanoparticles, such as the cross-nucleation of polymorphs ${ }^{22}$ or the fate of crystallization seeds during crystal growth. ${ }^{23}$

As another example, we study the structural transformations that $\mathrm{CsPbBr}_{3}$ perovskite nanocrystals ${ }^{24,25}$ undergo at elevated temperatures. The perovskite nanocrystal in Figure $4 \mathrm{~g}$ features a single crystalline domain highlighted in red. Heating the crystal with a $500 \mu$ s laser pulse $(1 \mathrm{~mW})$ renders it amorphous (Figure $4 \mathrm{i}$ ), a process that is known to be associated with the degradation of the performance of perovskite nanocrystals in optoelectronic applications and that hinders their use in highpower devices. $^{26} \mathrm{~A}$ snapshot taken during laser irradiation provides a subparticle picture of the mechanism involved, revealing that amorphization is initiated at the crystal boundaries and propagates inward (Figure 4h). We estimate that the amorphous domain expands with a speed of $35 \mathrm{~nm} /$ $\mathrm{ms}$ along the long axis of the crystal and with $5-12 \mathrm{~nm} / \mathrm{ms}$ along its short axis. These observations suggest that interfaces play an important role in the high-temperature amorphization of perovskite nanocrystals and that their modification may provide a means to improve temperature stability.

\section{CONCLUSION}

In conclusion, we demonstrate both static and dynamic atomic-resolution imaging with intense, high-brightness electron pulses from a field emitter. We show that such electron pulses enable high-resolution imaging in the presence of large amounts of drift. For example, if $0.2 \AA$ drift per frame are acceptable, then even a staggering drift rate of $40 \mathrm{~nm} / \mathrm{s}$ can be tolerated if one images with $500 \mu$ s electron pulses, as we have done here. This particularly stands to benefit the field of in situ electron microscopy, where drift presents a fundamental challenge $^{1}$ but may also be useful for any kind of highresolution imaging affected by beam induced drift, such as cryo-electron microscopy. Atomic-resolution single-shot imaging with microsecond time resolution will enable the study of a wide range of irreversible atomic-scale processes. Of particular interest are the dynamics of proteins whose relevant motions frequently occur on the microsecond to millisecond time scale. $^{27}$ Our experiments suggest that single-shot imaging of proteins in liquid in the presence of negative stain will be possible. ${ }^{14}$ For unstained samples, it may be necessary to increase the pulse duration or image stroboscopically. Finally, we note that a particularly appealing feature of our method is that switching the microscope to pulsed operation can be done on the fly and without changing alignment. ${ }^{1}$

\section{METHODS}

Time-Resolved Transmission Electron Microscopy. Experiments were performed with a modified JEOL 2010F transmission electron microscope that we have previously described. ${ }^{19}$ Intense, high-brightness electron pulses of microsecond duration are generated with a Schottky field emission gun operating at $200 \mathrm{kV}$ accelerating voltage. For the duration of the electron pulse, the emission current is boosted by illuminating the emitter tip with an intense microsecond laser pulse. $^{15}$ To this end, the output of a continuous laser $(532 \mathrm{~nm}$, $1.5 \mathrm{~W}$, Coherent Verdi) is chopped into microsecond pulses with an acousto-optic modulator (AA Optoelectronic, $50 \mathrm{~ns}$ rise time). The laser beam is then directed onto the emitter tip 
Crystallization of an individual lead nanoparticle
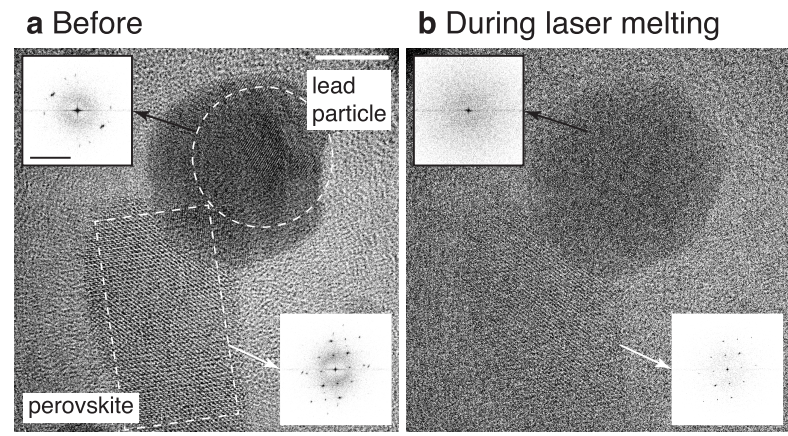

c After

d Before

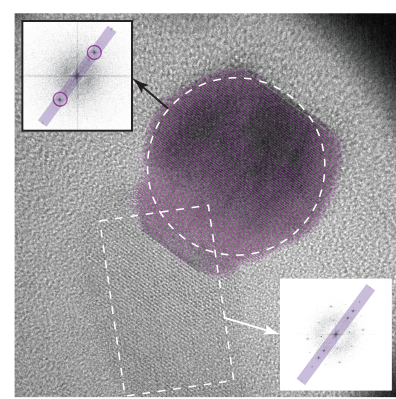

e +550 us (crystallization)

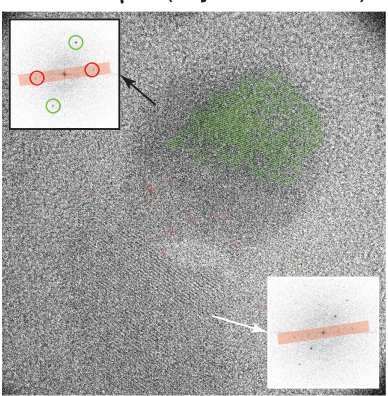

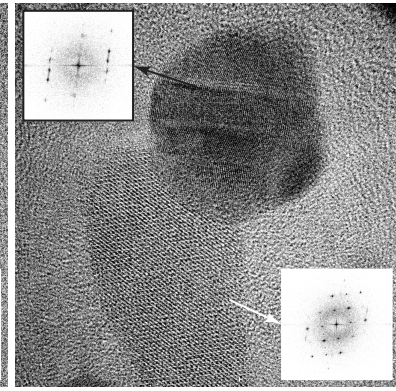

f After

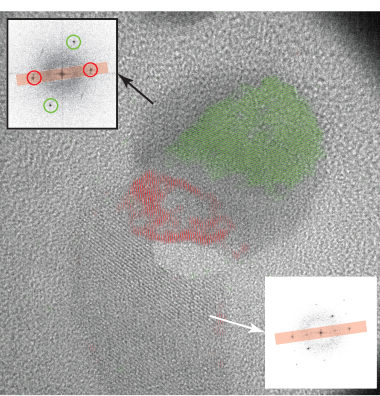

\section{Amorphization of a perovskite nanocrystal}

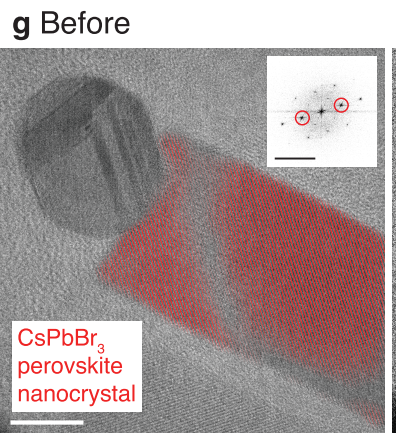

h $+300 \mu s$

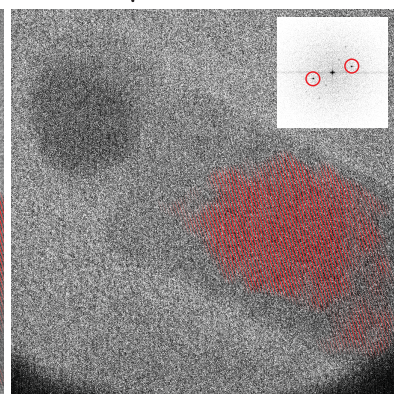

i After

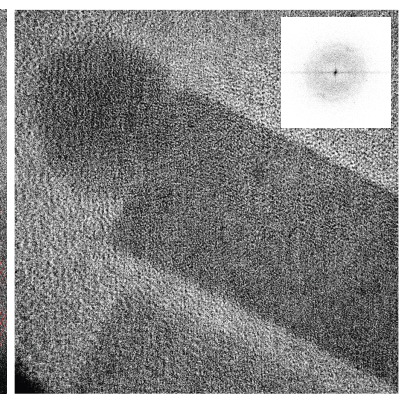

Figure 4. Single-shot imaging of atomic-scale transformations of nanoparticles. (a-f) Competing crystallization pathways of an individual lead nanoparticle. The lead particle (marked with circle in a) is melted in situ with a laser pulse $(500 \mu \mathrm{s}, 1 \mathrm{~mW})$ with the adjacent perovskite nanocrystal (rectangle) providing a nucleation seed for the subsequent crystallization process. A snapshot recorded during laser irradiation (b) reveals that the lead particle is completely melted, while the perovskite remains crystalline (100 $\mu$ s electron pulse, $200 \mu$ s before the end of the laser pulse). After the laser pulse, the lead particle cools and recrystallizes in a different configuration $(c)$. The insets show diffractograms of both particles. (d-f) The melting and recrystallization process is repeated $(500 \mu$ s laser pulse, $2 \mathrm{~mW})$, creating another configuration of the lead particle with several crystalline domains (f). Colored overlays highlight domains of different orientation and are obtained by Fourier filtering the images with the reflections selected that are circled in the diffractograms in the insets. A snapshot taken during the crystallization process (e, $550 \mu \mathrm{s}$ after the end of the laser pulse) reveals that the formation of the domain highlighted in green precedes that of the domain highlighted in red. Colored bars in the diffractograms highlight sets of reflections of the lead particle and the perovskite that are parallel to each other, suggesting that the perovskite acted as a nucleation seed in the crystallization process. Scale bars, $10 \mathrm{~nm}$ and $5 \mathrm{~nm}^{-1}$. $(\mathrm{g}-\mathrm{i})$ Amorphization of a perovskite nanocrystal under laser heating. The $\mathrm{CsPBBr}_{3}$ nanocrystal in panel gis rendered amorphous after illumination with a $500 \mu$ s laser pulse of $1 \mathrm{~mW}$ power (i), as evident from the diffractogram of the crystal in the inset. A snapshot recorded after $300 \mu$ s of laser irradiation (h) reveals that amorphization is initiated at the crystal boundaries ( $100 \mu$ s electron pulse). The red overlay highlights crystalline domains and is obtained by Fourier filtering the images with the reflections selected that are circled in the diffractogram in the inset. Scale bars, $10 \mathrm{~nm}$ and $5 \mathrm{~nm}^{-1}$.

(Figure 1a,b) where it is focused to a spot size of $17 \mu \mathrm{m}$ fwhm. Laser illumination temporarily heats the emitter tip to an extreme temperature of $2500 \mathrm{~K}$, almost quadrupling the emission current and increasing it to near its limit without damaging the emitter. ${ }^{15}$ The boosted electron beam is then chopped into pulses of variable duration by means of an electrostatic deflector that we installed between the electron gun and the microscope column (Figure 1a,b) and that consists of two stainless steel plates $(2 \mathrm{~mm}$ distance, $11 \mathrm{~mm}$ height, and $8 \mathrm{~mm}$ width). By applying a $400 \mathrm{~V}$ pulse to one of the plates with a fast high-voltage switch (Behlke), the beam is swept over a $300 \mu \mathrm{m}$ aperture located at a distance of about 10 $\mathrm{cm}$ from the deflector (Figure 1a,b). We can thus obtain laserboosted electron pulses of microsecond duration, and even pulses as short as $100 \mathrm{~ns}$. In order to induce sample drift or trigger dynamics, the sample is illuminating with the modulated output of a $405 \mathrm{~nm}$ diode laser (Cobolt, $2.5 \mathrm{~ns}$ 
rise/fall time), which is focused to a spot size of $34 \mu \mathrm{m}$ fwhm, as measured with a knife-edge scan at the sample position.

Determination of the Brightness of the LaserBoosted Electron Pulses. We measure the brightness of the laser-boosted electron pulses with the electron beam focused in the sample plane. Under such conditions, the brightness $B$ can be conveniently determined as the instantaneous probe current $\mathrm{Ne} / \Delta t$ per surface area element $\pi r^{2}$ and solid angle $\pi \alpha^{2}$

$$
B=\frac{\frac{N e}{\Delta t}}{\pi^{2} r^{2} \alpha^{2}}
$$

where $N$ is the number of electrons per pulse, $e$ is the electron charge, $\Delta t$ is the pulse duration, $r$ is the spot radius, and $\alpha$ is the convergence semiangle of the beam. ${ }^{19,28}$ Laser-boosted electron pulses were generated at $10 \mathrm{~Hz}$ repetition rate by irradiating the emitter tip with $100 \mu$ s laser pulses and using the deflectors to cut out $\Delta t=10 \mu \mathrm{s}$ long pulses from the most intense part of the laser-boosted electron beam. For each laser power, we recorded an image of the focused electron beam (spot size 1, alpha 3, condenser lens aperture number 3[50 $\mu \mathrm{m}$ diameter]) from which we determined the number of electrons per pulse $N$ as well as the fwhm diameter $2 r$ of the beam. Figure S1a shows that both the number of electrons and the beam diameter increase linearly with laser power. In contrast, the convergence semiangle of $\alpha=6.45 \mathrm{mrad}$, as determined from a diffraction pattern of the focused beam, is independent of laser power. With these quantities in hand, we calculate the instantaneous brightness of the electron pulses. Figures 1e and S1b show that the brightness initially increases linearly with laser power but then levels off as electron-electron interactions in the beam become more frequent. ${ }^{17,19}$ At a laser power of $0.64 \mathrm{~W}$, the brightness reaches $4.4 \times 10^{12} \mathrm{~A} / \mathrm{m}^{2}$ sr, about 1.7 times the value without boost laser.

Drift-Corrected Imaging with Microsecond Electron Pulses. For the experiments in Figure 2, reproducible amounts of drift were induced by illuminating the sample with a continuous laser beam. In Figure 2a,c, the laser power was held at $0.31 \mathrm{~mW}$ and linearly ramped to $2.6 \mathrm{~mW}$ during image acquisition (about $1 \mathrm{~s}$ total exposure time), resulting in an average sample drift velocity of $9 \mathrm{~nm} / \mathrm{s}$. In Figure $2 \mathrm{e}, \mathrm{g}$, the laser power was set to $0.26 \mathrm{~mW}$ and ramped to $0.8 \mathrm{~mW}$, resulting in a drift of $3 \mathrm{~nm} / \mathrm{s}$. All images in Figure 2 were recorded as $1 \mathrm{~s}$ movies (full-frame) of 25 frames. While the images in Figure 2a,e were acquired with a continuous electron beam and a dose rate of 9 electrons/pixel/s, those in Figure $2 \mathrm{c}$, g were recorded with $480 \mu$ s electron pulses (one pulse per movie frame) and the same total electron dose. We note that for pulsed illumination, the electron beam is more converged than in the images acquired with a continuous electron beam.

In order to ensure a fair comparison between the driftcorrected images recorded with a continuous electron beam and those acquired with electron pulses, the following procedure was followed. First, both movies were cropped to show exactly the same area of the sample, so that the effectiveness of the drift correction algorithm would not be affected by the visibility of different features in the two movies. The movie frames were then aligned with cisTEM $^{20}$ to determine the drift path of the sample. Since the drift paths of both movies differed slightly at the beginning and at the end, the initial and final frames were discarded, leaving only 10 frames in the micrographs of Figure 2a,c and 14 frames in the micrographs of Figure 2e,g. Finally, these remaining frames were again aligned with cisTEM to yield the images shown in Figure 2.

Atomic-Resolution Single-Shot Imaging. Single-shot experiments with laser-boosted electron pulses are performed with the electron beam almost fully converged on the sample and the illuminated area of the sample barely larger than the object under observation. In the micrographs of bacteriophage $\mathrm{T} 4$ in Figure 3c,d, the condenser lens stigmators were used to adjust the beam shape to the dimensions of the object. The micrographs in Figure 3a,c were obtained by recording 15 and 20 single-shot images, respectively, which were then driftcorrected and averaged.

In situ Growth of Perovskite Nanoparticles and In Situ Synthesis of Lead Nanoparticles. For the experiment on the high-temperature amorphization of perovskite nanocrystals (Figure $4 \mathrm{~g}-\mathrm{i}$ ), a $\mathrm{Cs} \mathrm{PbBr}_{3}$ perovskite nanocrystal of suitable dimensions was grown in situ. We laser evaporate an ensemble of $\mathrm{CsPbBr}_{3}$ perovskite quantum dots (Sigma-Aldrich) that had been deposited on a graphene oxide on lacey carbon specimen grid. At a distance from the laser focus where the temperature of the specimen grid is lower, the vapor condenses, and perovskite nanocrystals with a characteristic rectangular outline grow, such as the one in Figure $4 \mathrm{~g}$. Our in situ growth of perovskite nanoparticles resembles various vapor-phase epitaxy protocols that have previously been described. ${ }^{29}$

The lead nanoparticle in Figure $4 \mathrm{a}-\mathrm{f}$ was formed in situ as a side product of laser irradiation of $\mathrm{CsPbBr}_{3}$ perovskite quantum dots. The identity of the particle is confirmed by the diffractograms of its various configurations that we created by recrystallizing the particle in situ. Synthesizing the lead particle in situ avoids the formation of an oxide layer on its surface.

\section{ASSOCIATED CONTENT}

\section{(s) Supporting Information}

The Supporting Information is available free of charge at https://pubs.acs.org/doi/10.1021/acs.nanolett.0c04184.

Detailed methods, Figures S1-S4 (PDF)

\section{AUTHOR INFORMATION}

\section{Corresponding Author}

Ulrich J. Lorenz - Laboratory of Molecular Nanodynamics, École Polytechnique Fédérale de Lausanne, 1015 Lausanne, Switzerland; 이이.org/0000-0002-8869-5999; Email: ulrich.lorenz@epfl.ch

\section{Authors}

Pavel K. Olshin - Laboratory of Molecular Nanodynamics, École Polytechnique Fédérale de Lausanne, 1015 Lausanne, Switzerland

Gabriele Bongiovanni - Laboratory of Molecular Nanodynamics, École Polytechnique Fédérale de Lausanne, 1015 Lausanne, Switzerland

Marcel Drabbels - Laboratory of Molecular Nanodynamics, École Polytechnique Fédérale de Lausanne, 1015 Lausanne, Switzerland

Complete contact information is available at:

https://pubs.acs.org/10.1021/acs.nanolett.0c04184

\section{Author Contributions}

†P.K.O. and G.B. contributed equally. 


\section{Author Contributions}

U.J.L. conceived the research. M.D. and U.J.L. designed the experiment, G.B. and P.K.O. performed the experiments and analyzed data. U.J.L. wrote the manuscript with contribution of all authors.

\section{Notes}

The authors declare no competing financial interest.

\section{ACKNOWLEDGMENTS}

This work was supported by the ERC Starting Grant 759145 and by the Swiss National Science Foundation Grants PP00P2 163681 and 206021 183295. The authors would like to thank D. Demurtas for providing negatively stained samples of Bacteriophage T4.

\section{REFERENCES}

(1) Taheri, M. L.; Stach, E. A.; Arslan, I.; Crozier, P. A.; Kabius, B. C.; LaGrange, T.; Minor, A. M.; Takeda, S.; Tanase, M.; Wagner, J. B.; Sharma, R. Current Status and Future Directions for in Situ Transmission Electron Microscopy. Ultramicroscopy 2016, 170, 8695.

(2) Cheng, Y.; Grigorieff, N.; Penczek, P. A.; Walz, T. A Primer to Single-Particle Cryo-Electron Microscopy. Cell 2015, 161 (3), 438449.

(3) Westenfelder, B.; Meyer, J. C.; Biskupek, J.; Algara-Siller, G.; Lechner, L. G.; Kusterer, J.; Kaiser, U.; Krill, C. E.; Kohn, E.; Scholz, F. Graphene-Based Sample Supports for in Situ High-Resolution TEM Electrical Investigations. J. Phys. D: Appl. Phys. 2011, 44, 055502.

(4) Zeng, Z.; Liang, W.-I.; Liao, H.-G.; Xin, H. L.; Chu, Y.-H.; Zheng, H. Visualization of Electrode-Electrolyte Interfaces in $\mathrm{LiPF}_{6} /$ EC/DEC Electrolyte for Lithium Ion Batteries via in Situ TEM. Nano Lett. 2014, 14, 1745-1750.

(5) Liu, Z.; Monclús, M. A.; Yang, L. W.; Castillo-Rodríguez, M.; Molina-Aldareguía, J. M.; Llorca, J. Tensile Deformation and Fracture Mechanisms of $\mathrm{Cu} / \mathrm{Nb}$ Nanolaminates Studied by in Situ TEM Mechanical Tests. Extreme Mech. Lett. 2018, 25, 60-65.

(6) Foster, D. M.; Pavloudis, Th; Kioseoglou, J.; Palmer, R. E. Atomic-Resolution Imaging of Surface and Core Melting in Individual Size-Selected Au Nanoclusters on Carbon. Nat. Commun. 2019, 10, 2583.

(7) Voss, J. M.; Olshin, P. K.; Charbonnier, R.; Drabbels, M.; Lorenz, U. J. In Situ Observation of Coulomb Fission of Individual Plasmonic Nanoparticles. ACS Nano 2019, 13, 12445-12451.

(8) Zewail, A. H. Four-Dimensional Electron Microscopy. Science 2010, 328, 187-193.

(9) Browning, N. D.; Bonds, M. A.; Campbell, G. H.; Evans, J. E.; LaGrange, T.; Jungjohann, K. L.; Masiel, D. J.; McKeown, J.; Mehraeen, S.; Reed, B. W.; Santala, M. Recent Developments in Dynamic Transmission Electron Microscopy. Curr. Opin. Solid State Mater. Sci. 2012, 16, 23-30.

(10) Feist, A.; Bach, N.; Rubiano da Silva, N.; Danz, T.; Möller, M.; Priebe, K. E.; Domröse, T.; Gatzmann, J. G.; Rost, S.; Schauss, J.; Strauch, S.; Bormann, R.; Sivis, M.; Schäfer, S.; Ropers, C. Ultrafast Transmission Electron Microscopy Using a Laser-Driven Field Emitter: Femtosecond Resolution with a High Coherence Electron Beam. Ultramicroscopy 2017, 176, 63-73.

(11) Picher, M.; Bücker, K.; LaGrange, T.; Banhart, F. Imaging and Electron Energy-Loss Spectroscopy Using Single Nanosecond Electron Pulses. Ultramicroscopy 2018, 188, 41-47.

(12) Voisin, T.; Grapes, M. D.; Li, T. T.; Santala, M. K.; Zhang, Y.; Ligda, J. P.; Lorenzo, N. J.; Schuster, B. E.; Campbell, G. H.; Weihs, T. P. In Situ TEM Observations of High-Strain-Rate Deformation and Fracture in Pure Copper. Mater. Today 2020, 33, 10-16.

(13) Evans, J. E.; Browning, N. D. Enabling Direct Nanoscale Observations of Biological Reactions with Dynamic TEM. Microscopy 2013, 62, 147-156.
(14) Zhang, L.; Hoogenboom, J. P.; Cook, B.; Kruit, P. Photoemission Sources and Beam Blankers for Ultrafast Electron Microscopy. Struct. Dyn. 2019, 6, 051501.

(15) Bongiovanni, G.; Olshin, P. K.; Drabbels, M.; Lorenz, U. J. Intense Microsecond Electron Pulses from a Schottky Emitter. Appl. Phys. Lett. 2020, 116, 234103.

(16) van Veen, A. H. V.; Hagen, C. W.; Barth, J. E.; Kruit, P. Reduced Brightness of the ZrO/W Schottky Electron Emitter. J. Vac. Sci. Technol., B: Microelectron. Process. Phenom. 2001, 19, 2038-2044.

(17) Cook, B.; Verduin, T.; Hagen, C. W.; Kruit, P. Brightness Limitations of Cold Field Emitters Caused by Coulomb Interactions. J. Vac. Sci. Technol., B: Nanotechnol. Microelectron.: Mater., Process., Meas., Phenom. 2010, 28, C6c74-C6c79.

(18) Tiemeijer, P. C.; Bischoff, M.; Freitag, B.; Kisielowski, C. Using a Monochromator to Improve the Resolution in TEM to below $0.5 \AA$. Part I: Creating Highly Coherent Monochromated Illumination. Ultramicroscopy 2012, 114, 72-81.

(19) Olshin, P. K.; Drabbels, M.; Lorenz, U. J. Characterization of a Time-Resolved Electron Microscope with a Schottky Field Emission Gun. Struct. Dyn. 2020, 7, 054304.

(20) Grant, T.; Rohou, A.; Grigorieff, N. CisTEM, User-Friendly Software for Single-Particle Image Processing. eLife 2018, 7, No. e35383.

(21) Kostyuchenko, V. A.; Chipman, P. R.; Leiman, P. G.; Arisaka, F.; Mesyanzhinov, V. V.; Rossmann, M. G. The Tail Structure of Bacteriophage T4 and Its Mechanism of Contraction. Nat. Struct. Mol. Biol. 2005, 12, 810-813.

(22) Desgranges, C.; Delhommelle, J. Molecular Mechanism for the Cross-Nucleation between Polymorphs. J. Am. Chem. Soc. 2006, 128, $10368-10369$.

(23) Allahyarov, E.; Sandomirski, K.; Egelhaaf, S. U.; Löwen, H. Crystallization Seeds Favour Crystallization Only during Initial Growth. Nat. Commun. 2015, 6, 7110.

(24) Protesescu, L.; Yakunin, S.; Bodnarchuk, M. I.; Krieg, F.; Caputo, R.; Hendon, C. H.; Yang, R. X.; Walsh, A.; Kovalenko, M. V. Nanocrystals of Cesium Lead Halide Perovskites $\left(\mathrm{CsPbX}_{3}, \mathrm{X}=\mathrm{Cl}, \mathrm{Br}\right.$, and I): Novel Optoelectronic Materials Showing Bright Emission with Wide Color Gamut. Nano Lett. 2015, 15, 3692-3696.

(25) Akkerman, Q. A.; Rainò, G.; Kovalenko, M. V.; Manna, L. Genesis, Challenges and Opportunities for Colloidal Lead Halide Perovskite Nanocrystals. Nat. Mater. 2018, 17, 394-405.

(26) Liao, M.; Shan, B.; Li, M. In Situ Raman Spectroscopic Studies of Thermal Stability of All- Inorganic Cesium Lead Halide $\left(\mathrm{CsPbX}_{3}\right.$, $\mathrm{X}=\mathrm{Cl}, \mathrm{Br}, \mathrm{I}$ ) Perovskite Nanocrystals. J. Phys. Chem. Lett. 2019, 10, $1217-1225$.

(27) Henzler-Wildman, K.; Kern, D. Dynamic Personalities of Proteins. Nature 2007, 450, 964-972.

(28) Williams, D.; Carter, B. Transmission Electron Microscopy, A Textbook for Materials Science, 2nd ed.; Springer Science Business Media: New York, 2009.

(29) Wang, Y.; Yang, F.; Li, X.; Ru, F.; Liu, P.; Wang, L.; Ji, W.; Xia, J.; Meng, X. Epitaxial Growth of Large-Scale Orthorhombic CsPbBr Perovskite Thin Films with Anisotropic Photoresponse Property. Adv. Funct. Mater. 2019, 29, 1904913. 